\title{
Comorbidity profiles and inpatient outcomes during hospitalization for heart failure: an analysis of the U.S. Nationwide inpatient sample
}

Christopher S Lee ${ }^{1 *}$, Christopher V Chien², Julie T Bidwell ${ }^{3}$, Jill M Gelow², Quin E Denfeld ${ }^{3}$, Ruth Masterson Creber ${ }^{4}$, Harleah G Buck ${ }^{5}$ and James O Mudd ${ }^{2}$

\begin{abstract}
Background: Treatment of heart failure (HF) is particularly complex in the presence of comorbidities. We sought to identify and associate comorbidity profiles with inpatient outcomes during HF hospitalizations.

Methods: Latent mixture modeling was used to identify common profiles of comorbidities during adult hospitalizations for HF from the 2009 Nationwide Inpatient Sample ( $n=192,327)$.

Results: Most discharges were characterized by "common" comorbidities. A "lifestyle" profile was characterized by a high prevalence of uncomplicated diabetes, hypertension, chronic pulmonary disorders and obesity. A "renal" profile had the highest prevalence of renal disease, complicated diabetes, and fluid and electrolyte imbalances. A "neurovascular" profile represented the highest prevalence of cerebrovascular disease, paralysis, myocardial infarction and peripheral vascular disease. Relative to the common profile, the lifestyle profile was associated with a $15 \%$ longer length of stay (LOS) and 12\% greater cost, the renal profile was associated with a $30 \%$ higher risk of death, 27\% longer LOS and 24\% greater cost, and the neurovascular profile was associated with a $45 \%$ higher risk of death, 34\% longer LOS and $37 \%$ greater cost (all $p<0.001$ ).

Conclusions: Comorbidity profiles are helpful in identifying adults at higher risk of death, longer length of stay, and accumulating greater costs during hospitalizations for HF.
\end{abstract}

Keywords: Heart failure, Comorbidity, Outcomes, Inpatient

\section{Background}

Management of the heterogeneous disorder of heart failure $(\mathrm{HF})$ is particularly complex in the presence of comorbidities [1-3]. Nearly $60 \%$ of adults with HF have five or more chronic comorbidities, a percentage that has increased dramatically over the last twenty years [4]. Both cardiovascular and non-cardiovascular comorbidities observed among adults with HF contribute to condition development, progression and prognosis [5-7]. Comorbidities in HF also vary in etiological association [8], with some conditions like myocardial infarction and peripheral vascular disease having risk factors in common, and others such

\footnotetext{
* Correspondence: leechri@ohsu.edu

'Oregon Health \& Science University School of Nursing and Knight Cardiovascular Institute, 3455 SW US Veterans Hospital Road, Portland, OR 97239-2941, USA

Full list of author information is available at the end of the article
}

as cerebrovascular disease and dementia having a direct causal link. Thus, although the influence of specific and/or the number of comorbidities are important to our understanding of HF complexity, identifying naturally occurring patterns among comorbidities may be more helpful in identifying subgroups of HF patients who are more vulnerable to poor outcomes and who would benefit from tailored management strategies $[9,10]$.

Accordingly, the primary aim of this study was to identify and characterize distinct, common, and naturallyoccurring profiles among chronic comorbidities in adult hospitalizations for HF. To quantify the clinical relevance of these findings, we also sought to quantify associations among observed comorbidity profiles and the inpatient outcomes of all-cause death, length-of-stay (LOS) and costs. 


\section{Methods}

Data from the 2009 Agency for Healthcare Research and Quality (AHRQ) Health Care Utilization Project Nationwide Inpatient Sample (NIS) were used for this study. The NIS is the largest publicly available all-payer inpatient care database in the U.S. The 2009 NIS contains all discharge data from a sampling frame of 4,390 hospitals located in 44 States, representing an approximate $20 \%$ stratified sample of U.S. community hospitals. This study was determined to be exempt from ethical review by the Oregon Health \& Science University institutional review board because all NIS data are de-identified and includes several other safeguards to protect the privacy of individual patients, physicians and hospitals. This study conforms to the principles of the Declaration of Helsinki. All hospital discharges were examined for the diagnosis of HF by identifying AHRQ clinical classification software [11] diagnosis category 108 (i.e. congestive HF, nonhypertensive (International Classification of Diseases, $9^{\text {th }}$ Edition, Clinical Modifications (ICD-9-CM) codes 398.91, $428.0 \quad 428.1 \quad 428.20428 .21$ 428.22428 .23428 .30428 .31428 .32428 .33428 .40428 .41 $428.42428 .43428 .9)$ ) [12] and additional ICD-9-CM codes indicative of HF (402.01, 402.11, 402.91, 404.01, $404.03,404.11,404.13,404.91,404.93)[13,14]$ as the principal diagnoses. In the NIS, the principal diagnosis was the condition established after study to be chiefly responsible for prompting the hospitalization. Hospitalizations for HF were also verified as being urgent or emergent (i.e. non-elective).

\section{Assessment of comorbidities}

In accordance with a common definition, comorbidities were considered coexisting conditions to HF, the index disorder under study [15]. AHRQ comorbidity software [16] was used to generate binary variables that identify 29 comorbidities in discharge records using ICD-9-CM codes. Seventeen Deyo-Charlson clinical comorbidities [17] were also assessed using ICD-9-CM codes. There was both overlap and additive value in comparing the AHRQ and Deyo-Charlson comorbidities. Hence, a complement of 25 comorbid illnesses from the AHRQ software (acquired immunodeficiency syndrome, alcoholism, deficiency anemia, rheumatoid arthritis/collagen vascular disease, chronic blood loss anemia, chronic pulmonary disease, coagulopathy, depression, diabetes, uncomplicated, diabetes with chronic complications, drug abuse, hypertension, hypothyroidism, lymphoma, fluid and electrolyte disorders, metastatic cancer, other neurological disorders, obesity, paralysis, peripheral vascular disorders, psychoses, pulmonary circulation disorders, solid tumor without metastasis, valvular disease and weight loss) and 7 comorbid illnesses from the Deyo-Charlson comorbidity measures (acute myocardial infarction, cerebrovascular disease, dementia, peptic ulcer disease, mild liver disease, renal disease and moderate/severe liver disease). The number of chronic comorbidities and Charlson Comorbidity Index categories of comorbid burden (low (1-2), medium (3-4), high (5 or more)) [18] were also computed for comparisons.

\section{Inpatient outcomes}

Death was defined in the NIS as in-hospital mortality, and in this paper, is reported as all-cause. Length-of-stay was calculated as the number of nights the patient remained in the hospital for a particular discharge. Length-of-stay in this analysis was all-cause. Total charges reported in the 2009 NIS were converted to costs using hospital-specific cost-to-charge ratios based on actual accounting reports from the Centers for Medicare and Medicaid Services. Costs, in \$U.S. 2009, reported in this paper were all-cause.

\section{Statistical analysis}

In accordance with the structure of the NIS database, the primary unit of analysis was hospitalization. Means and proportions with standard errors adjusted for discharge weights were used to describe population estimates derived from this sample. Latent class mixture modeling was used to identify distinct and common comorbidity profiles among binary categorical variables indicating the presence or absence of 32 complementary comorbidities (performed with Mplus v.6, Los Angeles, California). Latent class mixture model specification was based on procedures explicated by Ram and colleagues [19]. The Lo-Mendell-Rubin adjusted likelihood ratio test (LMRT) [20], convergence (entropy near 1.0), sufficient proportions of the sample in each profile, and posterior probabilities (average probability of belonging in "most likely" profile near 1.0) were used to compare alternative models (e.g. $k$ vs. $k-1$ profiles) $[21,22]$; discharge weights were incorporated into all latent class models. Observed profiles were labeled according to dominant and differentiating comorbidities. Differences in comorbidities among observed profiles were quantified using $x^{2}$ tests. Multinomial logistic regression models were generated to calculate marginal probabilities (range -1 to 1 , sum to zero) [23] of belonging to each profile based on single comorbid conditions.

Generalized linear modeling was used to quantify associations among observed comorbidity profiles and inpatient death (relative risk (RR)) length-of-stay (incident rate ratio (IRR) based on the negative binomial distribution corrected for over-dispersion) and inpatient costs (gamma distribution for relative differences, and Duan's smearing retransformation of log-transformed cost [24] to estimate absolute differences in raw 2009 U.S. dollars) (performed with Stata v.11MP, College Station, Texas). Associations between the number of comorbidities and Charlson categories of comorbid burden and inpatient outcomes also 
were generated for context and comparison. Discharge weights were applied in all generalized linear models, and all estimates were adjusted for age, gender, race, median income of the patient's zip code, primary expected healthcare payer, and weekend vs. weekday admissions, as well as hospital bed size (small, medium, or large) control (government or private, government/nonfederal (public), private/non-profit, private/investor owned, private), location (rural, urban), region (Northeast, Midwest, South, West) and teaching status (non-teaching, teaching).

\section{Results}

\section{Characteristics of the population}

The sample consisted of 192,327 hospitalizations (population estimate $=976,664)$ with HF listed as the principal diagnosis (Table 1). Approximately $51 \%$ of discharges were for women, and the average age was almost 73 years. Death occurred during just over $3 \%$ of hospitalizations, the average length-of-stay was 5.2 days (median $=4$ days), and the average inpatient cost was $\$ 11,313$ (Table 2). In total, there were 12,966 inpatient deaths, 2.2 million inpatient days, and $\$ 4.6$ billion in inpatients costs translating to population estimates of 30,798 deaths, 5.1 million inpatient days, and a total of $\$ 10.3$ billion in inpatient costs.

\section{Number of comorbid illnesses, Charlson categories and inpatient outcomes}

The most prevalent comorbidities in this sample were hypertension, renal disease, chronic pulmonary disease, uncomplicated diabetes mellitus, and deficiency anemia (Figure 1). The average number of comorbid illnesses was 7.5 , and the vast majority of discharges $(82.4 \%)$ were for patients with a high Charlson comorbidity category (Table 2). There was a relatively small, but positive influence of the number of comorbidities on the risk of inpatient death $(\mathrm{RR}=1.03,95 \%$ confidence interval $(\mathrm{CI})=1.02-1.04)$, length-of-stay (IRR $=1.05,95 \% \mathrm{CI}=1.04-1.05)$, and inpatient cost (relative increase $=5.4 \%, 95 \% \mathrm{CI}=5.1 \%-5.6 \%)($ all $\mathrm{p}<$ 0.0001 ). Only $0.3 \%$ of discharges were for adults with a low Charlson comorbidity category - a subgroup too small for effective comparisons. Discharges for adults with a high vs. a medium Charlson comorbidity category, however, were associated with a greater risk of inpatient death $(R R=1.16$ (95\% CI $=1.08-1.25)$, longer length of stay $(\mathrm{IRR}=1.15$ (95\% CI $=1.13-1.17$ ), and greater inpatient cost (relative increase $=12.5 \%, 95 \% \mathrm{CI}=10.3 \%-14.7 \%)($ all $\mathrm{p}<0.0001)$.

\section{Identifying profiles of comorbidity}

Among the 32 conditions considered, four distinct comorbidity profiles were identified (model entropy $=0.71$; LMRT $\left.=16,296 ; \mathrm{p}<1 \times 10^{-7}\right)$. The largest profile $(\mathrm{n}=89,518$ (46.7\%), Figure 2-A), which was labeled as "common," included HF discharges with relatively few, but common comorbidities. There were 2 fewer comorbidities on average in the common profile than the other profiles. The common profile also had the lowest prevalence of cerebrovascular disease (0.8\%), myocardial infarction (13.9\%), peripheral vascular disorders (5.2\%), depression (7.5\%), renal disease (0.5\%), fluid and electrolyte disorders (20.8\%), hypertension (55.8\%), and obesity (9.3\%) compared with the other profiles. The prevalence of uncomplicated diabetes in the common profile was $27.4 \%$ and there were no cases of diabetes with chronic complications. The common profile, named in part because of the size of the profile and the relatively limited comorbid burden, served as a reference to which the other profiles could be compared.

A "lifestyle" profile ( $n=37,797$ (19.6\%), Figure 2-B) was characterized by a greater percentage of HF discharges with uncomplicated diabetes, hypertension, chronic pulmonary disorders and obesity than any other profile (all $\mathrm{p}<$ $\left.1 \times 10^{-7}\right)$. A "renal" profile $(n=57,228(29.6 \%)$, Figure $2-C)$ had a greater percentage of HF discharges with renal disease, diabetes with complications and fluid and electrolyte imbalances than any other profile (all $\mathrm{p}<1 \times 10^{-7}$ ). Finally, a "neurovascular" profile ( $n=7,784$ (4.1\%), Figure 2-D) had a greater percentage of HF hospitalizations with cerebrovascular disease, paralysis, myocardial infarction, peripheral vascular disease, neurological disorders and depression than any other profile (all $\mathrm{p}<1 \times 10^{-7}$ ).

Key profile-differentiating comorbidities are presented in Table 3 . In the presence of paralysis, the probability of belonging to the neurovascular comorbidity profile is $91.1 \%$ and the probability of belonging to the other profiles is dramatically reduced. Similarly, the presence of cerebrovascular disease is associated with a high probability (76.5\%) of belonging to the neurovascular profile and reduced probability of belonging to the other comorbidity profiles. The presence of diabetes with complications $(73.8 \%)$ and renal disease $(53.3 \%)$ increases the probability of belonging to the renal profile, and the presence of uncomplicated diabetes (57.3\%) and obesity (22.7\%) increase the probability of having the lifestyle profile of comorbidities.

\section{Profiles of comorbidity and inpatient outcomes}

Associations among observed comorbidity profiles and inpatient outcomes are presented in Table 4. Relative to the common comorbidity profile, inpatient death was $30.2 \%$ more likely for HF discharges fitting renal profile and 44.5\% more likely for HF discharges fitting the neurovascular profile (both $\mathrm{p}<1 \times 10^{-7}$ ). Relative to the common comorbidity profile, discharges within the lifestyle profile were associated with a $14.9 \%$ longer length of stay, discharges within the renal profile were associated with a 26.9\% longer length of stay, and HF discharges fitting the neurovascular profile were associated with a $33.6 \%$ longer length of stay (all $\mathrm{p}<1 \times 10^{-7}$ ). In raw days of length-ofstay, hospitalizations for patients fitting the lifestyle comorbidity profile were 0.68 days longer, hospitalizations 
Table 1 Characteristics of the sample $\left(n=192,327^{*}\right)$

\begin{tabular}{lcc}
\hline $\begin{array}{l}\text { Discharge and hospital } \\
\text { characteristics }\end{array}$ & $\begin{array}{c}\text { Estimate } \\
\text { (mean or proportion) }\end{array}$ & $\mathbf{9 5 \% ~ C l}$ \\
\hline Age (in years) & 72.88 & $(72.82-72.95)$ \\
$\begin{array}{l}\text { Gender (\% female) } \\
\text { Race/Ethnicity }{ }^{\dagger}\end{array}$ & $50.77 \%$ & $(50.55 \%-51.00 \%)$ \\
$\quad$ White & $68.02 \%$ & $(67.79 \%-68.24 \%)$ \\
Black & $18.98 \%$ & $(18.79 \%-19.17 \%)$ \\
Hispanic & $7.73 \%$ & $(7.61 \%-7.86 \%)$ \\
Asian or Pacific Islander & $1.79 \%$ & $(1.72 \%-1.85 \%)$ \\
Native American & $0.54 \%$ & $(0.51 \%-0.58 \%)$ \\
Other & $0.30 \%$ & $(0.29 \%-0.30 \%)$
\end{tabular}

Median zip code income national quartile

$\begin{array}{lll}\text { First } & 31.60 \% & (31.39 \%-31.81 \%) \\ \text { Second } & 27.00 \% & (26.80 \%-27.21 \%) \\ \text { Third } & 22.63 \% & (22.44 \%-22.82 \%) \\ \text { Fourth } & 18.77 \% & (18.59 \%-18.95 \%)\end{array}$

Primary healthcare payer

Medicare
Medicaid
Private insurance
Self-pay
No charge
Other

Admission on a weekend

Hospital bed size

Small

Large

Control

Government or private
Government, nonfederal
Private, non-profit
Private, investor owned
Private
Location
Rural
Urban
Region
Northeast
Midwest
South
West

\section{$(7.87 \%-8.11 \%)$}

$(74.11 \%-74.51 \%$

(12.09\%-12.39\%)

(3.46\%-3.63\%)

(0.30\%-0.36\%)

(1.54\%-1.65\%)

(23.23\%-23.60\%)

(13.12\%-13.43\%)

(23.68\%-24.07\%)

(62.63\%-63.07\%)

(59.31\%-59.75\%)

$(7.07 \%-7.30 \%)$

(18.20\%-18.55\%)

(10.58\%-10.86\%)

(4.10\%-4.28\%)

(14.76\%-15.08\%)

(84.92\%-85.23\%)

$(22.16 \%-22.54 \%)$

$(23.21 \%-23.59 \%)$

(39.15\%-39.59\%)

(14.72\%-15.03\%)
Table 1 Characteristics of the sample $\left(n=192,327^{*}\right)$ (Continued)

\begin{tabular}{lll}
\hline Teaching status & & \\
Non-teaching & $58.78 \%$ & $(58.56 \%-59.00 \%)$ \\
Teaching & $41.22 \%$ & $(41.00 \%-41.44 \%)$
\end{tabular}

"Estimates generated using discharge weights (population estimate $n=976,664$ ).

${ }^{+}$data missing for $14.3 \%$ of discharges. $\mathrm{Cl}$, confidence interval.

for patients fitting the renal profile were 1.26 days longer, and hospitalizations for patients fitting the neurovascular profile were 1.57 days longer on average than HF discharges fitting the common profile (all $\mathrm{p}<1 \times 10^{-7}$ ). Relative to the common comorbidity profile, discharges within the lifestyle profile were associated with $11.9 \%$ greater inpatient costs, those fitting the renal profile were associated with $23.5 \%$ greater inpatient costs, and those within the neurovascular profile were associated with $36.7 \%$ greater inpatient costs (all $\mathrm{p}<1 \times 10^{-7}$ ). In raw 2009 US dollars, hospitalizations for patients fitting the lifestyle profile cost $\$ 1,422.36$ more, hospitalizations for patients fitting the renal profile cost $\$ 2,199.26$ more, and hospitalizations for patients fitting the neurovascular profile cost $\$ 2,809.39$ more on average compared with HF discharges fitting the common profile.

\section{Discussion}

Management of HF is complicated by prevalent cardiovascular and non-cardiovascular comorbidities [25]. In this nationally-representative inpatient sample of 192,327 adult hospitalizations with a principal diagnosis of HF, there was an average of 7.5 comorbidities and the vast majority of hospitalizations $(82.4 \%)$ were categorized as having a high Charlson comorbid burden. The main finding of this study is the identification of four distinct and naturally occurring

Table 2 Comorbidities and outcome characteristics ( $\mathrm{n}=192,327^{*}$ )

\begin{tabular}{lcc}
\hline $\begin{array}{l}\text { Comorbidities } \\
\text { and outcomes }\end{array}$ & $\begin{array}{c}\text { Estimate } \\
\text { (mean or proportion) }\end{array}$ & $\mathbf{9 5 \% ~ C l}$ \\
\hline $\begin{array}{l}\text { Number of } \\
\text { comorbidities }\end{array}$ & 7.54 & $(7.53-7.55)$ \\
$\begin{array}{l}\text { Charlson comorbidity } \\
\text { category }\end{array}$ & & \\
$\quad$ Low (1-2) & $0.29 \%$ & $(0.26 \%-0.31 \%)$ \\
$\quad$ Medium (3-4) & $17.29 \%$ & $(17.12 \%-17.46 \%)$ \\
$\quad$ High (5+) & $82.42 \%$ & $(82.25 \%-82.60 \%)$ \\
Inpatient death & $3.15 \%$ & $(3.08 \%-3.23 \%)$ \\
Inpatient length & 5.22 & $(5.20-5.25)$ \\
of stay (days) & & $(\$ 11,233.77-\$ 11,329.80)$ \\
Inpatient cost & $\$ 11,313.28$ & \\
(2009 $\$$ US) & &
\end{tabular}

"Estimates generated using discharge weights (population estimate $n=976,664$ ). Abbreviations: $\mathrm{Cl}$, confidence interval. 


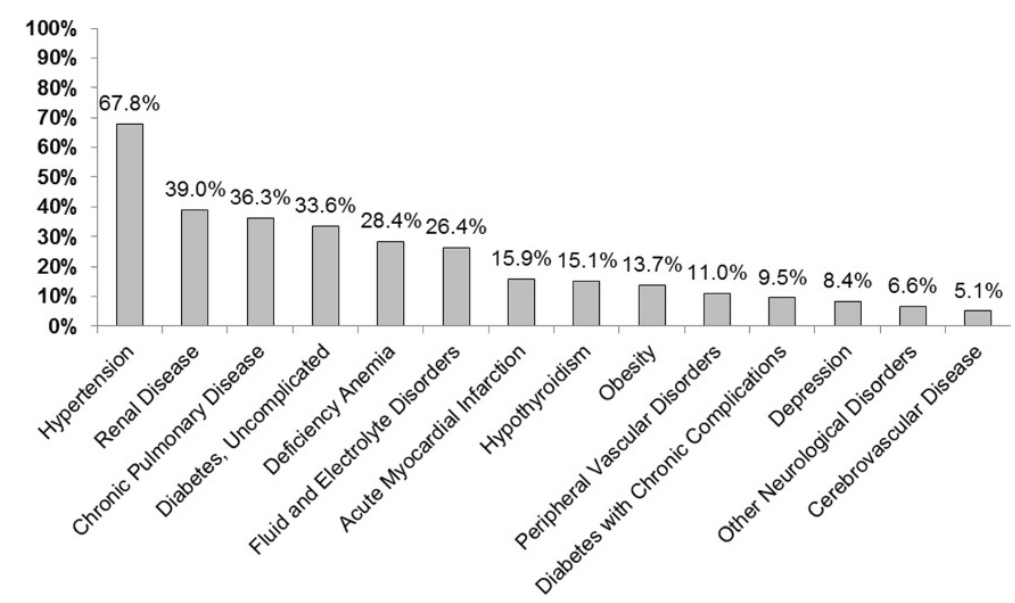

Figure 1 Prevalence of comorbidities in adult hospitalizations with a principal diagnosis of heart failure. Prevalence estimates were generated using discharge weights $(n=192,327$; population estimate $n=976,664)$. Only conditions with a prevalence of $5 \%$ or greater are displayed for economy of presentation.

comorbidity profiles that were associated with significant differences in the all-cause inpatient outcomes of death, length-of-stay and cost. Relative to the $46.7 \%$ of hospitalizations with a "common" comorbidity profile, the $19.6 \%$ of HF hospitalizations fitting a "lifestyle" profile had longer length-of-stay and greater costs, and the $29.6 \%$ of HF hospitalizations within a "renal" comorbidity profile and the $4.1 \%$ of HF hospitalizations with a "neurovascular" comorbidity profile were associated with higher risk of inpatient death, longer length-of-stay and greater costs.

Similar to the findings of other studies, we observed a direct relationship between the number of comorbidities [26-28] as well as categories of Charlson comorbid burden [29] and clinical outcomes among adults with HF. Specifically, we observed an adjusted 3\%-5\% increase in the risk of death, extension in length-of-stay and accrual of inpatient costs for additional comorbidities. The number of comorbidities and the proportion of discharges associated with 5 or more comorbidities in this sample are greater than prior reports in HF $[4,28]$, which can be explained, at least in part, by our inclusion of 32 comorbidities that were of both cardiovascular and non-cardiovascular origin. An extreme minority of hospitalizations in this sample involved patients with a low comorbid burden; thus, we compared hospitalizations fitting a moderate and high Charlson comorbid risk. High Charlson comorbid burden was associated with $12 \%-16 \%$ increase in the risk of death, extension in length-of-stay and accrual of inpatient costs compared with hospitalizations for adults with a moderate comorbid burden. In sum, our findings provide further evidence in support of both the number of comorbidities and categorization of comorbid burden as having a significant influence on inpatient outcomes in HF.

There is elegance in the simplicity of counting comorbidities, considering thresholds that represent complex comorbidity (i.e. 5 or more), and weighing counts of comorbidities based on established associations with 1-year mortality (i.e. Charlson). A major limitation to these approaches to comorbidities in HF, however, is the inability to consider how some comorbidities are naturally independent, others have common risk factors and/or pathogenic pathways, and others still have direct causation [8]. Moreover, single comorbidity inventories often omit key conditions that are highly-prevalent in HF (e.g. the Charlson excludes hypertension, and the AHRQ comorbidities do not include myocardial infarction), and ineffectively capture condition severity (e.g. the Charlson does not differentiate diabetes with and without complications, and the AHRQ comorbidities do not differentiate mild from moderate to severe liver disease). Thus, our finding of four distinct, naturally-occurring profiles among comorbidities in adult HF hospitalizations builds upon prior research that has involved single comorbidity inventories or considered all comorbidities as being etiologically and statistically independent.

The largest comorbidity profile we observed involved the fewest and common comorbidities. In fact, the only differentiating factors to identity patients fitting this profile were prevalent HF comorbidities (e.g. hypertension, chronic pulmonary disease, and uncomplicated diabetes) and the absence of renal disease, which had an overall prevalence of $39 \%$ but only affected $0.5 \%$ of discharges in this profile. Based on this sample, there is an estimated 455,851 hospitalizations in the U.S. for HF patients fitting this comorbidity profile annually, and they experience the most favorable inpatient outcomes. The next hazardous profile we observed was differentiated by comorbidities that are linked to lifestyle, including 100\% prevalence of uncomplicated diabetes, $84 \%$ prevalence of hypertension, $40 \%$ prevalence of chronic pulmonary disease, and $27 \%$ 


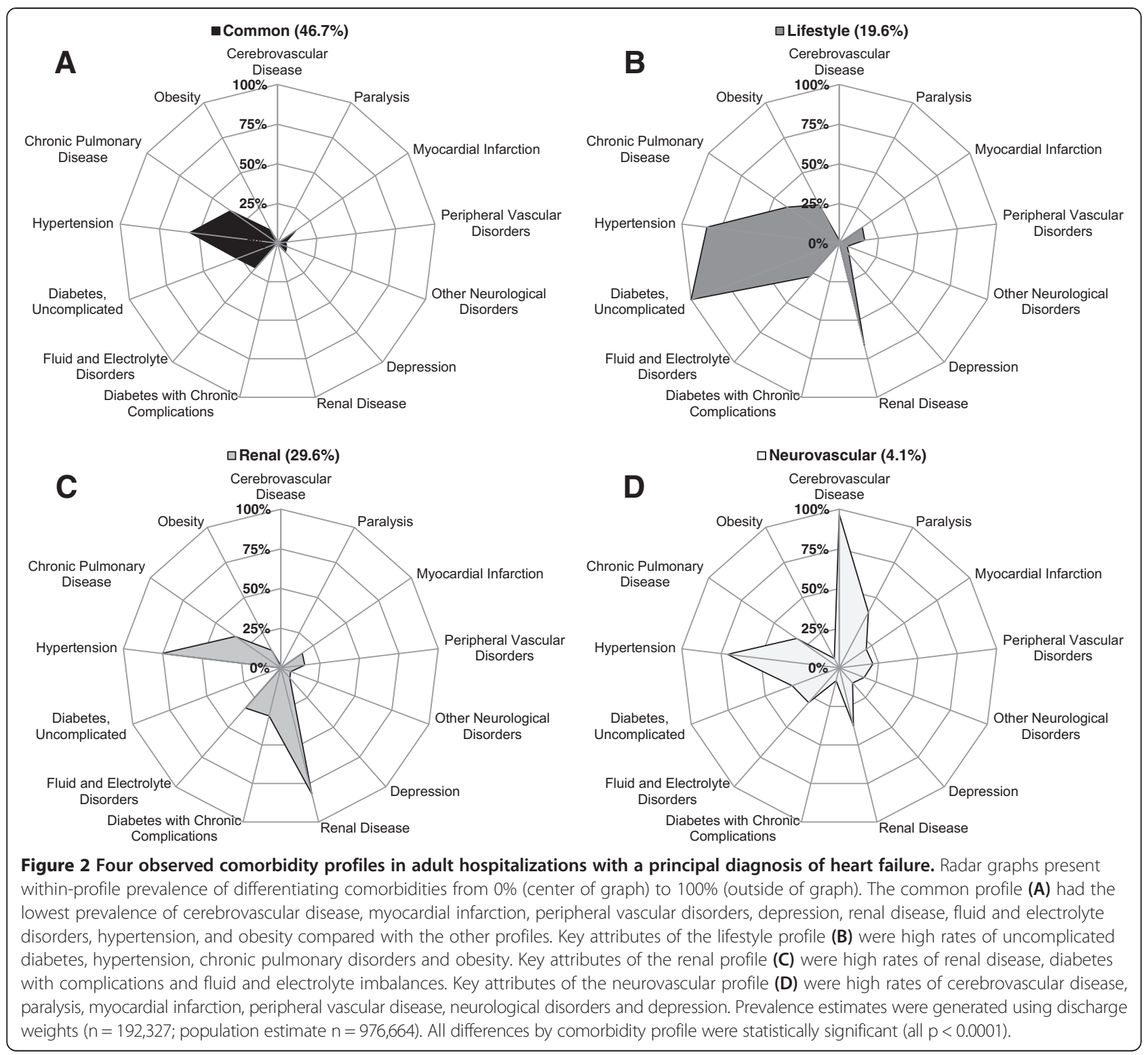

Table 3 Key differentiating comorbidities: marginal probabilities ( $n=192,327^{*}$ )

\begin{tabular}{|c|c|c|c|c|}
\hline Comorbidity & $\begin{array}{c}\text { Common } n=89,518^{+} \\
(46.7 \%)\end{array}$ & $\begin{array}{c}\text { Lifestyle } n=37,797^{\dagger} \\
(19.6 \%)\end{array}$ & $\begin{array}{c}\text { Renal } n=57,228^{\dagger} \\
(29.6 \%)\end{array}$ & $\begin{array}{c}\text { Neurovascular } \\
n=7,784^{\dagger}(4.1 \%)\end{array}$ \\
\hline Paralysis & -0.422 & -0.187 & -0.302 & 0.911 \\
\hline Cerebrovascular disease & -0.417 & -0.129 & -0.220 & 0.765 \\
\hline Diabetes with complications & -0.515 & -0.217 & 0.738 & -0.006 \\
\hline Renal disease & -0.755 & 0.224 & 0.533 & -0.002 \\
\hline Diabetes, uncomplicated & -0.142 & 0.573 & -0.428 & -0.003 \\
\hline Obesity & -0.175 & 0.227 & -0.028 & -0.024 \\
\hline
\end{tabular}

All marginal probabilities were statistically significant (all $p<0.0001$ ). Marginal effects in probability scale sum to zero for each comorbidity, with values closest to \pm 1.0 indicating the strongest differentiation.

"Estimates generated using discharge weights (population estimate $n=976,664$ ).

${ }^{\dagger}$ Population estimates for totals of discharges fitting the common, lifestyle, renal and neurovascular comorbid illness profiles were 455851, 191595, 289613, and 39604, respectively. 
Table 4 Adjusted differences in inpatient outcomes by comorbidity profile $\left(n=192,327^{*}\right)$

\begin{tabular}{|c|c|c|c|c|c|c|}
\hline \multirow[b]{2}{*}{ Comorbidity profile } & \multicolumn{2}{|c|}{ Inpatient death } & \multicolumn{2}{|c|}{ Length-of-stay } & \multicolumn{2}{|c|}{ Inpatient cost } \\
\hline & RR $(95 \% \mathrm{Cl})$ & p-value & IRR $(95 \% \mathrm{Cl})$ & p-value & Relative cost $(95 \% \mathrm{Cl})$ & p-value \\
\hline Lifestyle $^{\dagger}$ & $1.023(0.945-1.108)$ & 0.573 & $1.149(1.135-1.163)$ & $<0.001$ & $111.9 \%(110.0 \%-113.9 \%)$ & $<0.001$ \\
\hline Renal $^{\dagger}$ & $1.302(1.223-1.387)$ & $<0.001$ & $1.269(1.254-1.284)$ & $<0.001$ & $123.5 \%(121.5 \%-125.4 \%)$ & $<0.001$ \\
\hline Neurovascular ${ }^{\dagger}$ & $1.445(1.280-1.631)$ & $<0.001$ & $1.336(1.291-1.381)$ & $<0.001$ & $136.7 \%(131.3 \%-142.3 \%)$ & $<0.001$ \\
\hline
\end{tabular}

Note: All estimates are adjusted for age, gender, race, median income of the patient's zip code, primary healthcare payer, and weekend vs. weekday admissions, as well as hospital bed size (small, medium, or large) control (government or private, government/nonfederal (public), private/non-profit, private/investor owned,

private), location (rural, urban), region (Northeast, Midwest, South, West) and teaching status (non-teaching, teaching).

${ }^{*}$ Estimates generated using discharge weights (population estimate $n=976,664$ ).

${ }^{\dagger}$ Relative to the "common" comorbidity profile.

Abbreviations: $\mathrm{Cl}$, confidence interval calculated from weighted standard errors; IRR, incident rate ratio; RR, relative risk.

prevalence of obesity. The triad of diabetes, hypertension and obesity is well-recognized as integral characteristics of metabolic syndrome [30], and majority of chronic pulmonary disease is thought to be attributable to cigarette smoking [31]. There is an estimated 191,595 annual hospitalizations for HF patients fitting this lifestyle comorbidity profile that is associated with longer length-of-stay and greater inpatient cost. Fitting the lifestyle profile on discharges for HF should serve as a red flag for greater healthcare utilization, and interventions to reduce the risk of poor inpatient outcomes for this profile should be tailored to improving lifestyle in general in addition to treating specific comorbidities. Since hypertension and diabetes mellitus were prominent features of both the common and lifestyle comorbidity profiles, dietary modifications and aerobic exercise are two specific lifestyle recommendations that may be helpful in the reduction of cardiovascular risk in these two groups [32]. It is also known that hypertension and obesity are more prevalent among HF patients with preserved versus reduced ejection fraction [33]; thus, interventions to reduce the risk of poor clinical outcomes may be further tailored by HF type within the observed comorbidity profiles.

The next hazardous profile we observed was centered on a high prevalence of renal disease $(82 \%)$ and diabetes with complications (31\%). The combination of renal disease and diabetes has been shown by others to be associated greater risk of death and hospitalization in HF [34]. Over 3/4 of HF discharges within this renal profile also had hypertension and slightly more than $1 / 3$ had fluid and electrolyte disorders. Diabetes and hypertension are widely recognized as the leading causes of renal disease [35], and cardiorenal syndrome [36] is a challenging condition to manage particularly during acute hospitalization for HF [37]. Given the nature of these data, it is not possible to understand the temporal and/or causal nature of relationships among the key differentiating comorbidities in the renal or other profiles. But, HF hospitalizations for patients fitting this profile were associated with a $30 \%$ increase in the risk of inpatient death and $27 \%$ and $24 \%$ increases in length-of-stay and inpatient costs, respectively and there is an estimated 289,613 annual HF discharges in the U.S. for patients fitting this comorbidity profile.
The most hazardous comorbidity profile identified was differentiated from the others by extremely high rates of cerebrovascular disease, and the highest prevalence of paralysis, myocardial infarction, peripheral vascular disorders, neurologic disorders and depression. Peripheral vascular disease is often paired with comorbid cerebrovascular disease [38], and is a determinant of worse outcomes in patients with myocardial infarction [39]. There also is a link between both cerebrovascular disease [40] and myocardial infarction [41] and depression. Thus, end-organ damage of global vascular disease appeared to be central to this profile. Based on this sample there are more than 39,000 HF hospitalizations for patients fitting this neurovascular comorbidity profile in the U.S. annually; there was a $45 \%$ increase in the risk of inpatient death, 34\% increase in length-of-stay and $37 \%$ increase in inpatient cost associated with discharges for HF patients fitting this profile. Patients fitting the renal or neurovascular profiles upon admission for $\mathrm{HF}$ should be assumed to require greater healthcare utilization and should be enrolled in disease management and care transition programs to mitigate the overall impact on global healthcare resources and improve patients' quality-of-life.

There are several existing schemes for classifying the risk of poor inpatient outcomes in HF. For example, Fonarow and colleagues determined that blood urea nitrogen, admission systolic blood pressure, and serum creatinine were predictive of inpatient mortality among adults admitted for decompensated HF [42]. Coronary artery disease, renal insufficiency and diabetes were more prevalent in the highversus the low-risk groups in that study. As an additional example, Peterson and group determined that age, systolic blood pressure, blood urea nitrogen, heart rate, serum sodium, coexisting chronic obstructive pulmonary disease, and race were predictive of inpatient mortality among adults admitted for decompensated HF [43]. Thus, the incorporation of coexisting comorbidities into inpatient prognostic models is not new. But, what our findings add to extant knowledge about the influence of comorbidities on inpatient outcomes is a new approach the naturally-occurring clusters of prevalent and coexisting that complicate HF admissions and are associated with significant differences in inpatient outcomes. 


\section{Strengths and limitations}

The present study has strengths and limitations. Using the NIS as a nationally-representative sample of patients admitted for the principal diagnosis of HF and applying sampling weights allows us to generalize estimates for comorbidity prevalence, composition of comorbidity profiles and inpatient outcomes that are generalizable to a much larger population than the sample studied. Tradeoffs for this strength are the limitations of using hospitalization (and not patient) as the unit of analysis, and that national representativeness does not translate to generalizability to all persons with HF. There are also challenges to using ICD-9-CM codes for the identification of HF and comorbidities alike. The ICD-9-CM codes chosen for this study, however, are highly predictive of HF when compared with clinical criteria [12], and are established codes for performance measures in HF [13]. Like most research involving administrative databases, there may have been under-reporting of chronic comorbidities in the NIS [44]. It is also likely that the comorbidity profiles we identified are dynamic rather than fixed; thus, these profiles are likely to evolve over time along with the progression of both HF and concomitant comorbidities. We are also unable to distinguish casual relationships among comorbidities, those simple having risk factors in common and the potential pathological links to HF (e.g. complication of, predisposing factor for, or unrelated) in this single evaluation of administrative data; hence, that was not our goal.

We also did not differentiate between HF with preserved or unpreserved ejection fraction. There are important socio-demographic (e.g. older age and female gender), etiological (e.g. greater prevalence of hypertension and atrial fibrillation, and less frequent coronary artery disease) [45], and non-cardiovascular comorbidity differences (e.g. high prevalence of chronic lung, liver and gastric disorders) comparing HF patients with preserved versus reduced ejection [46]. Hence, it will be important to differentiate comorbidity profiles by groups of HF patients with preserved and reduced ejection fraction in future research. Because of the administrative nature of this data source, we were unable to incorporate granular clinical data (e.g. ejection fraction, which ventricles are involved, blood pressure, glomerular filtration rate, and functional performance measures). Hence, future research is warranted to examine the influence of comorbidity profiles along with clinical data including, but not limited to, a) the assessment of HF with preserved versus reduced ejection fraction, b) the natural history of comorbidities over time and how they relate to the evolution of HF, c) different manifestations of HF (e.g. left, right, or bi-ventricular dysfunction), and d) other prognostic clinical data.

\section{Conclusion}

Among a large sample of adult hospitalizations for HF, we observed four distinct comorbidity profiles that were associated with large and significant differences in the risk of inpatient death, length-of-stay and inpatient cost. Naturally-occurring patterns of cardiovascular and noncardiovascular comorbidities, not simply the number of comorbidities, should be the focus of future research and the target of future interventions aimed at reducing the risk of inpatient death and healthcare utilization.

\section{Abbreviations:}

AHRQ: Agency for Healthcare Research and Quality; Cl: Confidence interval; HF: Heart failure; ICD-9-CM: International Classification of Diseases, $9^{\text {th }}$ Edition, Clinical Modifications; IRR: Incident rate ratio; LMRT: Lo-Mendell-Rubin adjusted likelihood ratio test; LOS: Length of stay; NIS: Nationwide inpatient sample; RR: Relative risk.

\section{Competing interests}

The authors declare that they have no competing interests.

\section{Authors' contributions}

CSL conceived of the study, gained access to these data, performed all statistical analysis and led the development of the manuscript. CC, JMG, and JOM served as cardiovascular medicine experts, made substantive

improvements to the analysis and written manuscript, and took the lead on describing the clinical relevance of these findings. HGB served as an external expert on the comorbidities in heart failure and provided substantive feedback on the analysis and made major contributions to the writing of the manuscript. JTB, QED, and RMC were integrally involved with the statistical analysis and assisted with final model solutions and presentation of these data; they also made substantive contributions to the writing of the manuscript. All authors read and approved the final manuscript.

\section{Acknowledgements}

This work was supported in part by the Office of Research on Women's Health and the National Institute of Child Health and Human Development through the Oregon Building Interdisciplinary Research on Women's Health program (HD043488-08). The content is solely the responsibility of the authors and does not necessarily represent the official views of the Office of Research on Women's Health, the National Institute of Child Health and Human Development, or the National Institutes of Health.

\section{Author details}

${ }^{1}$ Oregon Health \& Science University School of Nursing and Knight Cardiovascular Institute, 3455 SW US Veterans Hospital Road, Portland, OR 97239-2941, USA. ${ }^{2}$ Oregon Health \& Science University Knight Cardiovascular Institute, 3181 S.W. Sam Jackson Park Rd, Portland, OR 97239, USA. ${ }^{3}$ Oregon Health \& Science University School of Nursing, 3455 SW US Veterans Hospital Road, Portland, OR 97239-2941, USA. ${ }^{4}$ University of Pennsylvania School of Nursing, 418 Curie Blvd., Philadelphia, PA 19104, USA. ${ }^{5}$ The Pennsylvania State University College of Nursing, 201 Health and Human Development East University Park, Philadelphia, PA 16802, USA.

Received: 25 March 2014 Accepted: 30 May 2014

Published: 5 June 2014

\section{References}

1. Jessup M, Abraham WT, Casey DE, Feldman AM, Francis GS, Ganiats TG, Konstam MA, Mancini DM, Rahko PS, Silver MA, Stevenson LW, Yancy CW: 2009 focused update: ACCF/AHA Guidelines for the Diagnosis and Management of Heart Failure in Adults: a report of the American College of Cardiology Foundation/American Heart Association Task Force on Practice Guidelines: developed in collaboration with the International Society for Heart and Lung Transplantation. Circulation 2009, 119(14):1977-2016. 
2. Murad K, Kitzman DW: Frailty and multiple comorbidities in the elderly patient with heart failure: implications for management. Heart Fail Rev 2012, 17(4-5):581-588.

3. Dahlstrom U: Frequent non-cardiac comorbidities in patients with chronic heart failure. Eur J Heart Fail 2005, 7(3):309-316.

4. Wong CY, Chaudhry SI, Desai MM, Krumholz HM: Trends in comorbidity, disability, and polypharmacy in heart failure. Am J Med 2011, 124(2):136-143.

5. Triposkiadis FK, Skoularigis J: Prevalence and importance of comorbidities in patients with heart failure. Curr Heart Fail Rep 2012, 9(4):354-362.

6. Lang CC, Mancini DM: Non-cardiac comorbidities in chronic heart failure. Heart 2007, 93(6):665-671.

7. Ather S, Chan W, Bozkurt B, Aguilar D, Ramasubbu K, Zachariah AA, Wehrens XH, Deswal A: Impact of noncardiac comorbidities on morbidity and mortality in a predominantly male population with heart failure and preserved versus reduced ejection fraction. J Am Coll Cardiol 2012, 59(11):998-1005.

8. Valderas JM, Starfield B, Sibbald B, Salisbury C, Roland M: Defining comorbidity: implications for understanding health and health services. Ann Fam Med 2009, 7(4):357-363.

9. Saczynski JS, Go AS, Magid DJ, Smith DH, McManus DD, Allen L, Ogarek J, Goldberg RJ, Gurwitz JH: Patterns of comorbidity in older adults with heart failure: the Cardiovascular Research Network PRESERVE study. J Am Geriatr Soc 2013, 61(1):26-33.

10. Sarkar IN, Chen ES: Determining compound comorbidities for heart failure from hospital discharge data. AMIA Annu Symp Proc 2012, 2012:809-818.

11. Agency for Healthcare Research and Quality Clinical Classifications Software (CCS) for ICD-9-CM; http://www.hcup-us.ahrq.gov/toolssoftware/ccs/ccs.jsp.

12. Lee DS, Donovan L, Austin PC, Gong Y, Liu PP, Rouleau JL, Tu JV: Comparison of coding of heart failure and comorbidities in administrative and clinical data for use in outcomes research. Med Care 2005, 43(2):182-188

13. Bonow RO, Bennett S, Casey DE Jr, Ganiats TG, Hlatky MA, Konstam MA, Lambrew CT, Normand SL, Pina IL, Radford MJ, Smith AL, Stevenson LW, Burke G, Eagle KA, Krumholz HM, Linderbaum J, Masoudi FA, Ritchie JL, Rumsfeld JS, Spertus JA: ACC/AHA Clinical Performance Measures for Adults with Chronic Heart Failure: a report of the American College of Cardiology/American Heart Association Task Force on Performance Measures (Writing Committee to Develop Heart Failure Clinical Performance Measures): endorsed by the Heart Failure Society of America. Circulation 2005, 112(12):1853-1887.

14. Blecker S, Paul M, Taksler G, Ogedegbe G, Katz S: Heart failure-associated hospitalizations in the United States. J Am Coll Cardio/ 2013, 61(12):1259-1267.

15. de Groot V, Beckerman H, Lankhorst GJ, Bouter LM: How to measure comorbidity. a critical review of available methods. J Clin Epidemiol 2003, 56(3):221-229.

16. Elixhauser A, Steiner $C$, Harris DR, Coffey RM: Comorbidity measures for use with administrative data. Med Care 1998, 36(1):8-27.

17. Deyo RA, Cherkin DC, Ciol MA: Adapting a clinical comorbidity index for use with ICD-9-CM administrative databases. J Clin Epidemiol 1992, 45(6):613-619.

18. Charlson ME, Pompei P, Ales KL, Mackenzie CR: A new method of classifying prognostic comorbidity in longitudinal studies: development and validation. J Chronic Dis 1987, 40(5):373-383.

19. Ram N, Grimm KJ: Methods and Measures: Growth mixture modeling: A method for identifying differences in longitudinal change among unobserved groups. Int J Behav Dev 2009, 33(6):565-576.

20. Lo Y, Mendell NR, Rubin DB: Testing the number of components in a normal mixture. Biometrika 2001, 88(3):767-778.

21. Jung T, Wickrama KA: An introduction to latent class growth analysis and growth mixture modeling. Soc Pers Compass 2008, 2(1):302-317.

22. Nylund $\mathrm{KL}$, Asparouhov T, Muthen B: Deciding on the number of classes in latent class analysis and growth mixture modeling: a monte carlo simulation study. Struct Equ Modeling 2007, 14(4):535-569.

23. Hedeker D: A mixed-effects multinomial logistic regression model. Stat Med 2003, 22(9):1433-1446.

24. Duan N: Smearing estimate: a nonparametric retransformation method. J Am Stat Assoc 1983, 78(3838):605-610.

25. Nagarajan $\mathrm{V}$, Tang WH: Management of comorbid conditions in heart failure: a review. Med Clin North Am 2012, 96(5):975-985.

26. Ahluwalia SC, Gross CP, Chaudhry SI, Ning YM, Leo-Summers L, Van Ness PH, Fried TR: Impact of comorbidity on mortality among older persons with advanced heart failure. J Gen Intern Med 2012, 27(5):513-519.

27. Senni M, Parrella P, De Maria R, Cottini C, Bohm M, Ponikowski P, Filippatos G, Tribouilloy C, Di Lenarda A, Oliva F, Pulignano G, Cicoira M, Nodari S, Porcu M,
Cioffi G, Gabrielli D, Parodi O, Ferrazzi P, Gavazzi A: Predicting heart failure outcome from cardiac and comorbid conditions: the 3C-HF score. Int J Cardiol 2013, 163(2):206-211.

28. Braunstein JB, Anderson GF, Gerstenblith G, Weller W, Niefeld M, Herbert R, Wu AW: Noncardiac comorbidity increases preventable hospitalizations and mortality among Medicare beneficiaries with chronic heart failure. J Am Coll Cardiol 2003, 42(7):1226-1233.

29. Testa G, Cacciatore F, Galizia G, Della-Morte D, Mazzella F, Russo S, Ferrara N, Rengo F, Abete P: Charlson Comorbidity Index does not predict long-term mortality in elderly subjects with chronic heart failure. Age Ageing 2009, 38(6):734-740

30. Alberti KG, Eckel RH, Grundy SM, Zimmet PZ, Cleeman Jl, Donato KA Fruchart JC, James WP, Loria CM, Smith SC Jr: Harmonizing the metabolic syndrome: a joint interim statement of the International Diabetes Federation Task Force on Epidemiology and Prevention; National Heart Lung, and Blood Institute; American Heart Association; World Heart Federation; International Atherosclerosis Society; and International Association for the Study of Obesity. Circulation 2009, 120(16):1640-1645.

31. Mannino DM, Braman S: The epidemiology and economics of chronic obstructive pulmonary disease. Proc Am Thorac Soc 2007, 4(7):502-506.

32. Eckel RH, Jakicic JM, Ard JD, Hubbard VS, de Jesus JM, Lee IM, Lichtenstein AH, Loria CM, Millen BE, Miller NH, Nonas CA, Sacks FM, Smith SC Jr, Svetkey LP, Wadden TW, Yanovski SZ: 2013 AHA/ACC Guideline on Lifestyle Management to Reduce Cardiovascular Risk: A Report of the American College of Cardiology/American Heart Association Task Force on Practice Guidelines. Circulation 2013, [Epub ahead of print].

33. Owan TE, Hodge DO, Herges RM, Jacobsen SJ, Roger VL, Redfield MM: Trends in prevalence and outcome of heart failure with preserved ejection fraction. N Engl J Med 2006, 355(3):251-259.

34. Ekundayo OJ, Muchimba M, Aban IB, Ritchie C, Campbell RC, Ahmed A: Multimorbidity due to diabetes mellitus and chronic kidney disease and outcomes in chronic heart failure. Am J Cardiol 2009, 103(1):88-92.

35. Solini A, Ferrannini E: Pathophysiology, prevention and management of chronic kidney disease in the hypertensive patient with diabetes mellitus. J Clin Hypertens (Greenwich) 2011, 13(4):252-257.

36. Ronco C, Haapio M, House AA, Anavekar N, Bellomo R: Cardiorenal syndrome. J Am Coll Cardiol 2008, 52(19):1527-1539.

37. Aronson D: Cardiorenal syndrome in acute decompensated heart failure. Expert Rev Cardiovasc Ther 2012, 10(2):177-189.

38. Golomb BA, Dang TT, Criqui MH: Peripheral arterial disease: morbidity and mortality implications. Circulation 2006, 114(7):688-699.

39. Inglis SC, Bebchuk J, Al-Suhaim SA, Case J, Pfeffer MA, Solomon SD, Hou YR, Pitt B, Dargie HJ, Ford I, Kjekshus J, Zannad F, Dickstein K, McMurray JJ: Peripheral artery disease and outcomes after myocardial infarction: An individual-patient meta-analysis of 28,771 patients in CAPRICORN, EPEHESUS, OPTIMAAL and VALIANT. Int J Cardio/ 2012, 168(2):1094-1101.

40. Steffens DC, Helms MJ, Krishnan KR, Burke GL: Cerebrovascular disease and depression symptoms in the cardiovascular health study. Stroke 1999, 30(10):2159-2166.

41. Echols MR, O'Connor CM: Depression after myocardial infarction. Curr Heart Fail Rep 2010, 7(4):185-193.

42. Fonarow GC, Adams KF Jr, Abraham WT, Yancy CW, Boscardin WJ: Risk stratification for in-hospital mortality in acutely decompensated heart failure: classification and regression tree analysis. JAMA 2005, 293(5):572-580.

43. Peterson PN, Rumsfeld JS, Liang L, Albert NM, Hernandez AF, Peterson ED, Fonarow GC, Masoudi FA: A validated risk score for in-hospital mortality in patients with heart failure from the American Heart Association get with the guidelines program. Circ Cardiovasc Qual Outcomes 2010, 3(1):25-32.

44. Corser W, Sikorskii A, Olomu A, Stommel M, Proden C, Holmes-Rovner M: Concordance between comorbidity data from patient self-report interviews and medical record documentation. BMC Health Serv Res 2008, 8:85.

45. Meta-analysis Global Group in Chronic Heart Failure: The survival of patients with heart failure with preserved or reduced left ventricular ejection fraction: an individual patient data meta-analysis. Eur Heart J 2012, 33(14):1750-1757.

46. Lam CS, Donal E, Kraigher-Krainer E, Vasan RS: Epidemiology and clinical course of heart failure with preserved ejection fraction. Eur J Heart Fail 2011, 13(1):18-28.

doi:10.1186/1471-2261-14-73

Cite this article as: Lee et al:: Comorbidity profiles and inpatient outcomes during hospitalization for heart failure: an analysis of the U.S. Nationwide inpatient sample. BMC Cardiovascular Disorders 2014 14:73. 